\title{
Brief communication: Structural monitoring for lifetime extension of offshore wind monopiles: can strain measurements at one level tell us everything?
}

\author{
Lisa Ziegler ${ }^{1,2}$, Ursula Smolka ${ }^{1}$, Nicolai Cosack ${ }^{1}$, and Michael Muskulus ${ }^{2}$ \\ ${ }^{1}$ Ramboll Wind, 20097 Hamburg, Germany \\ ${ }^{2}$ Department of Civil and Environmental Engineering, Norwegian University of Science and Technology \\ NTNU, 7491 Trondheim, Norway \\ Correspondence to: Lisa Ziegler (lisa.ziegler@ ramboll.com)
}

\author{
Received: 4 May 2017 - Discussion started: 12 May 2017 \\ Revised: 18 July 2017 - Accepted: 31 July 2017 - Published: 12 September 2017
}

\begin{abstract}
Operators need accurate knowledge on structural reserves to decide about lifetime extension of offshore wind turbines. Load monitoring enables us to directly compare design loads with real loading histories of the support structure in order to calculate its remaining useful lifetime. Monitoring of every hot spot is technically and financially not feasible. This paper presents a novel idea for load monitoring of monopiles. It requires strain measurements at only one level convenient for sensor installation, such as tower bottom. Measurements are converted into damage equivalent loads for $10 \mathrm{~min}$ time intervals. Damage equivalent loads are extrapolated to other locations of the structure with a simulation model and statistical algorithm. For this, structural loads at all locations of the monopile are calculated with aero-hydro-elastic software and updated finite element models. Damage equivalent loads at unmeasured locations are predicted from the simulation results with a $k$-nearest neighbor regression algorithm. The extrapolation was tested with numerical simulations of an $8 \mathrm{MW}$ offshore wind turbine. Results show that damage can be predicted with an error of 1-3\% if this is done conditional on mean wind speed, which is very promising. The load monitoring concept is simple, cheap and easy to implement. This makes it ideal for making decisions on lifetime extension of monopiles.
\end{abstract}

\section{Introduction}

Load monitoring of foundations for offshore wind turbines enables to reconstruct load histories that these structures experienced. The load history can be compared against design loads to calculate remaining useful lifetimes, which is essential for decisions on lifetime extension. Direct monitoring of every hot spot at the structure is impossible due to cost and access restrictions. Structural responses must be extrapolated from a limited set of sensors.

In 2016, $81 \%$ of offshore wind turbine foundations were monopiles (Ho and Mbistrova, 2017). Existing monitoring strategies for monopiles are based on physical models or artificial intelligence. Model-based time-domain algorithms require accelerometers and (partly) strain gauges at the structure. They try to reproduce the time history of dynamic response parameters, such as acceleration or strain, of the whole structure. This has been investigated for monopiles using Kalman filters (Maes et al., 2016; Fallais et al., 2016), joint input-state estimation (Maes et al., 2016), and modal expansion algorithms (Maes et al., 2016; Iliopoulos et al, 2016).

In many cases, the remaining useful lifetime can be assessed using accumulated cycles or equivalent loads. Detailed load time series are not required. This is exploited by artificial intelligence algorithms (e.g., neural networks) (Smolka and Cheng, 2013; Cosack, 2011). After being trained using measurement data from all hot spots, the algorithms deduce statistics of dynamic response parameters, such as equivalent loads, from standard signals (Smolka et al, 2014).

There is almost no experience with lifetime extension of offshore wind turbines yet. Vindeby, the first commercial 

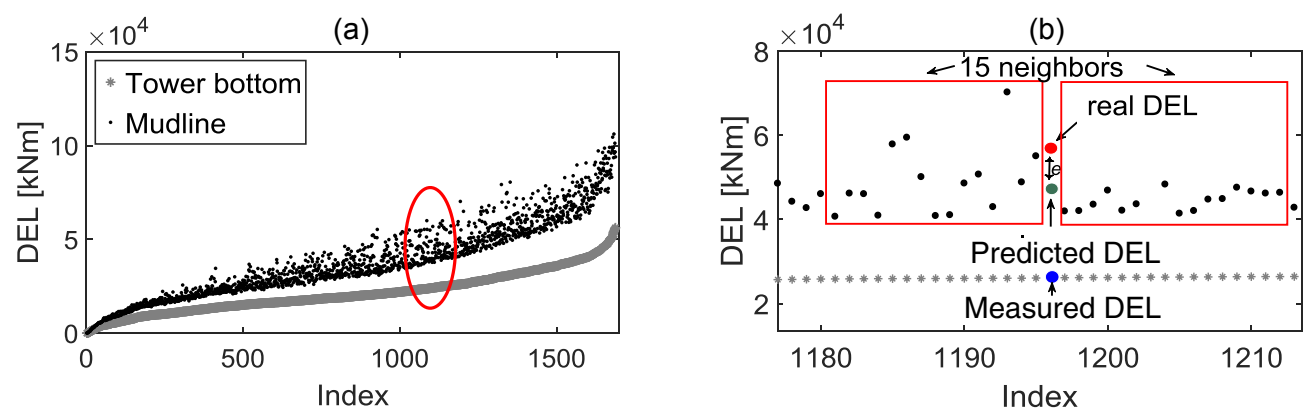

(c)

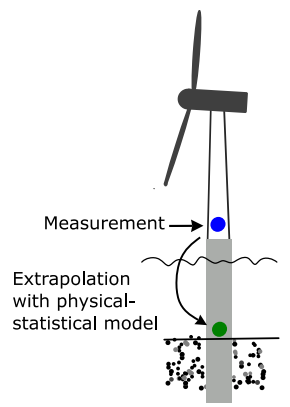

Figure 1. (a) DELs at tower bottom (T-DELs) sorted ascending for 1700 load cases. Corresponding DELs at mudline (M-DELs) are plotted as function of T-DELs. (b) Zoom into picture above. The M-DEL (green dot) corresponding to a measured T-DEL (blue dot) is estimated as mean value of 15 nearest neighbor values (from simulations) on each side. It deviates from the real M-DEL with an estimation error $e$. (c) Schematic setup of load monitoring concept. Measured T-DELs are extrapolated to other locations.

offshore wind farm installed in 1991, was decommissioned recently after 25 years of operation. Other existing structures, e.g., bridges, offshore oil platforms, and lately onshore wind turbines, have dealt with lifetime extension for multiple years already. Lifetime extension assessments and decision making in the oil and gas industry are discussed by Ersdal and Hörnlund (2008). Jackets for oil platforms are redundant structures where even the loss of some members is often within acceptable limits of probability of failure. Lifetime assessments focus on detection of fatigue cracks in combination with fracture mechanics analyses. For offshore wind monopiles, however, Ziegler and Muskulus (2016a) have shown that the probability of detecting decisive fatigue cracks for lifetime extension of monopiles is small as the crack growth is expected to progress fast in the circumferential welds of these structures once it reaches a certain size. The authors conclude that numerical fatigue reassessment and structural monitoring is needed for lifetime extension decisions of monopiles. The state of the art of lifetime extension in the onshore wind industry is reviewed by Ziegler et al. (2017). Typically, lifetime extension assessments have an analytical and/or practical part. The analytical part is a numerical fatigue reassessment where structural loading is recalculated with updated design models and certain assumptions (mainly environmental and operational conditions) (Ziegler and Muskulus, 2016b). Drawbacks are that long-term measurements of some environmental conditions, such as turbulence intensity, are often not available or expensive to obtain. The practical part is on-site inspections, which would be possible but expensive due to offshore risks (Ziegler and Muskulus, 2016a). Load monitoring will be useful for lifetime extension; however, operators are still reluctant due to associated costs.

Therefore, we developed a novel load monitoring concept that requires only minimal sensor placement. Load measurements at tower bottom are transformed into damage equivalent loads and extrapolated to other hot spots. This novel idea is presented in Sect. 2. Performance of the algorithm is discussed in Sect. 3 and concluded in Sect. 4.

\section{Methodology}

The methodology presented here requires to measure loads at only one location of the structure where installation and maintenance is convenient, such as near tower bottom. This information is used to predict damage equivalent loads (DELs) at all relevant hot spots of the monopile. Figure 1b illustrates the setup.

DEL is defined as the single-amplitude load (or stress) range that causes the same amount of damage over a reference number of cycles $N_{k}$ as the variable-amplitude load (or stress) time series $S_{i}$ with the corresponding number of cycles $N_{i}$ (see Eq. 1). Here $n$ is the number of stress ranges, and $m$ is the inverse slope of the considered stress-cycle curve (SN-curve) according to DNVGL (2016). Further information on DELs can be found in Cosack (2011).

$\mathrm{DEL}=\left(\sum_{i=1}^{n} \frac{N_{i}}{N_{k}} S_{i}^{m}\right)^{\frac{1}{m}}$

The methodology consists of the following steps:

1. The finite-element (FE) model of the monopile from the design phase is updated (e.g., with short-term on-site measurements) to ensure consistent dynamic behavior.

2. Aero-hydro-elastic simulations are performed with the updated model and an extended design basis. Simulation outputs are $10 \mathrm{~min}$ load (or stress) time series at the measurement location and at all locations of interest. Rainflow counting is performed on these time series and DELs are calculated for all hot spots of interest.

3. The transfer function between hot spots and measurement location are calibrated using simulated DELs. Details on the model are given in Sect. 2.1. 

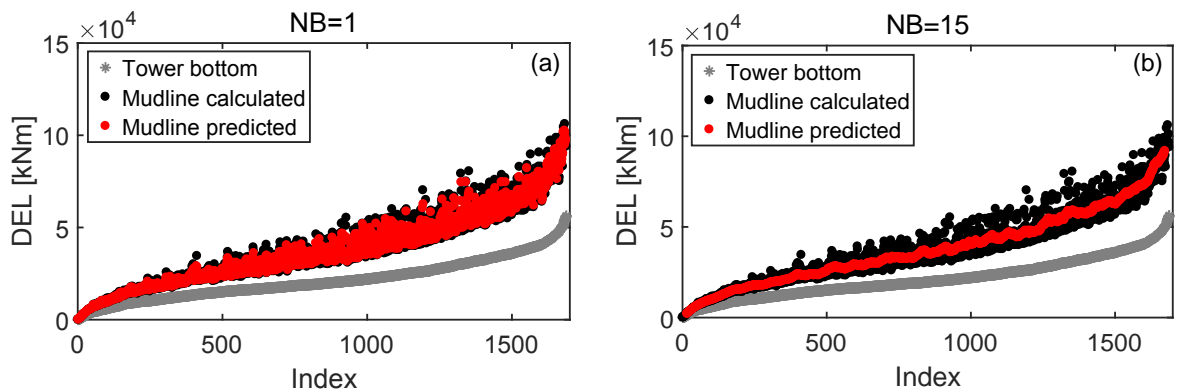

Figure 2. Simulated (black dots) and extrapolated M-DELs (red dots) considering one neighbor (a) and 15 neighbors (b) applying leaveone-out cross-validation.
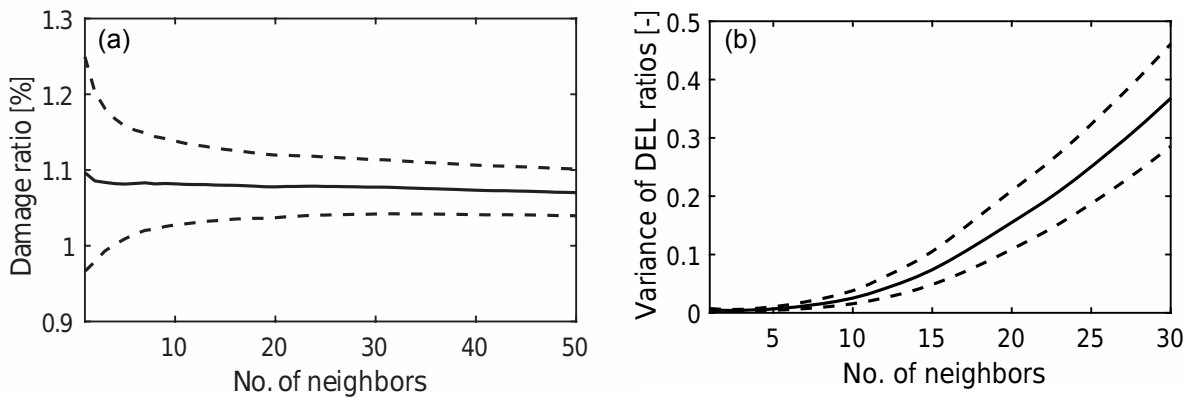

Figure 3. (a) Estimation error of lifetime damage as function of number of neighbors considered in the extrapolation algorithm. (b) Variance of individual DEL ratios as function of number of neighbors. DEL ratios are calculated as "extrapolated M-DEL/simulated M-DEL".

4. The load measurements are converted into $10 \mathrm{~min}$ DELs. The extrapolation model is used to predict the DELs at other locations of the structure.

5. Calculation of accumulated fatigue damage $D$ at desired locations and remaining useful lifetime with Eqs. (2) and (3). Here $a$ is the value of the SN curve at $N_{k}$ cycles and $t_{\mathrm{op}}$ is the number of years the wind turbine has operated already.

$$
\begin{gathered}
D=\frac{\mathrm{DEL}^{m} N_{k}}{a} \\
\mathrm{RUL}=\frac{t_{\mathrm{op}}}{D}-t_{\mathrm{op}}
\end{gathered}
$$

\subsection{Extrapolation model}

The relationship between DELs at tower bottom (T-DELs) and other locations of the monopile is assumed to be well defined. The extrapolation to DELs at mudline (M-DELs) is investigated as an example in the following. In Fig. 1a T-DELs from aero-hydro-elastic simulations are plotted in ascending order for 1700 load cases. The corresponding M-DELs are shown as black dots. Each load case has different inputs in terms of mean wind speed, turbulence intensity, sea state, wind and wave directionality, and operational condition.

Figure 1a shows evidence for the existence of a welldefined lower envelope for M-DELs. Scatter of M-DELs above this curve is limited. The highest M-DEL is only a factor of 2.3 higher than the lowest M-DEL for similar T-DELs.

The observed small scatter of M-DELs enables to use a simple statistical model for T-DEL extrapolation (see Fig. 1):

1. Sort a measured T-DEL into the array of simulated TDELs.

2. Select a number of simulated T-DELs close to the measured T-DEL value (nearest neighbors).

3. Predict desired M-DEL as mean or a weighted mean of the simulated M-DEL values corresponding to the nearest neighbor simulated T-DELs. Weighting can be done with occurrence probability of simulation load cases, when statistics from the site are available.

This methodology is an application of the $k$-nearest neighbors regression algorithm from machine learning (Murphy, 2012).

\subsection{Accuracy and choice of neighbors}

The accuracy of the extrapolation model is validated against simulation data here, as measurements were not available at this project stage. We use leave-one-out cross validation to assess the performance: one simulation result is dismissed (considered as "measured T-DEL"), the correspond- 
ing M-DEL is extrapolated with the remaining simulations, and then compared with the value known from simulations.

Figure 2 shows extrapolated M-DELs in red plotted over simulated M-DELs for one neighbor (a) and for 15 neighbors on each side (b). An increase in number of neighbors causes smoothing of extrapolation results. The number of neighbors should be chosen so that damage at the end of service life is predicted best.

Figure 3 a shows a measure of the estimation error of accumulated damage as a function of number of neighbors (solid line). Damage ratios are calculated as fraction "extrapolated damage/simulated damage". The dashed line indicates extrapolation uncertainty. It represents the deviation of damage from desired results when the standard error of the mean of the neighbors $\triangle \mathrm{MDEL}$ is added to the mean value during $\mathrm{M}$ DEL extrapolation (see Eq. 4). $\sigma$ is the standard deviation of the sample of considered neighbors, $n_{\mathrm{b}}$ is number of neighbors.

$$
\triangle \mathrm{MDEL}=\frac{\sigma}{\sqrt{n_{\mathrm{b}}}}
$$

The damage ratio converges to 1.08 after four neighbors in this example (design basis, no binning, mean of neighbors see Sect. 3). The deviation of lifetime damage decreases for an increasing number of neighbors at the beginning. Beyond four neighbors, the extrapolation accuracy seems insensitive to the number of neighbors used. The true value of 1.0 is inside the interval of 2 standard deviations (not plotted). The variance of the extrapolation error of individual DEL values increases with the number of neighbors (see Fig. 3b).

Available data from the turbine control and performance monitoring system (SCADA) can provide additional information for improving the extrapolation. Potentially relevant parameters are average wind speed, wind direction, and operational condition (power production or idling). To utilize this information, simulated DELs are binned according to these parameters. Only DELs that have similar conditions (i.e., are from the same bin) are considered as neighbors in the extrapolation.

\subsection{FE model updating}

The FE model of the monopile from the design phase must be updated before the extrapolation can be performed (see Sect. 2). The process of FE model updating should verify that the global dynamic behavior of the structure is captured correctly in the simulation model. Typical model updating techniques try to match natural frequencies, mode shapes, and damping. Operational modal analysis has been applied by Devriendt et al. (2014) to identify natural frequencies and damping of an offshore wind turbine using accelerometers distributed at tower and transition piece. Modern turbines are often equipped with accelerometers in the nacelle. Additional accelerometers at the tower or transition piece are not always present. Maes et al. (2016) showed that the first and second fore-aft and side-side natural frequencies of a monopile are identifiable from strain gauge measurements at the tower in operating conditions of the wind turbine by transforming strain time series into power spectral densities.

After identification of the relevant modal properties, a sensitivity analysis should reveal which parameters in the original design model are uncertain and influential on the mismatched modal properties. For the case of the monopile support structure, these parameters can be, for instance, soil properties, manufacturing tolerances, grouted connection (early designs of transition pieces) and secondary steel elements if omitted in the initial FE model. Several methods exist to update the FE model through minimization of an objective function addressing the selected parameters as described in the standard literature (e.g., Friswell and Mottershead, 1995). The updating procedure should be repeated in time to identify possible changes on natural frequencies of the structure. Such changes could occur, for instance, due to scour or soil stiffening over time. Future work with measurement data is necessary to address FE model updating based on strain measurements for a monopile and the sensitivity of the extrapolation algorithm to this.

\subsection{Simulation software}

The software used for load simulations were LACflex and ROSAP (in-house tools of Ramboll). LACflex is an aeroelastic software for time-domain analysis of wind turbines based on the solver FLEX 5 (Øye, 1999). ROSAP is a structural analysis program which Ramboll uses for design of offshore wind foundations. The detailed model of the monopile is reduced to a Craig-Bampton superelement including corresponding wave loads for accurate integration into LACflex. Response time series at tower bottom are imported into ROSAP to model hydrodynamic loading and structural response of the detailed FE model of monopile and transition piece (Passon and Branner, 2014; Passon, 2015). Design simulations of $10 \mathrm{~min}$ duration were performed for the fatigue load cases power production (DLC 1.2) and idling (DLC 6.4) according to IEC 61400-3 (IEC, 2009). Time series of the bending moment around a local axis at a single point of the circumferential of tower (near tower bottom) and monopile (near mudline) were extracted from the simulations. The point of the circumferential would be chosen identical to the location of the strain gauges in a practical application.

\section{Results and discussion}

\subsection{Case study}

Results are presented for a case study of a monopile in 30$40 \mathrm{~m}$ water depth supporting an $8 \mathrm{MW}$ wind turbine. Turbine and monopile were modeled in detail following industry state 

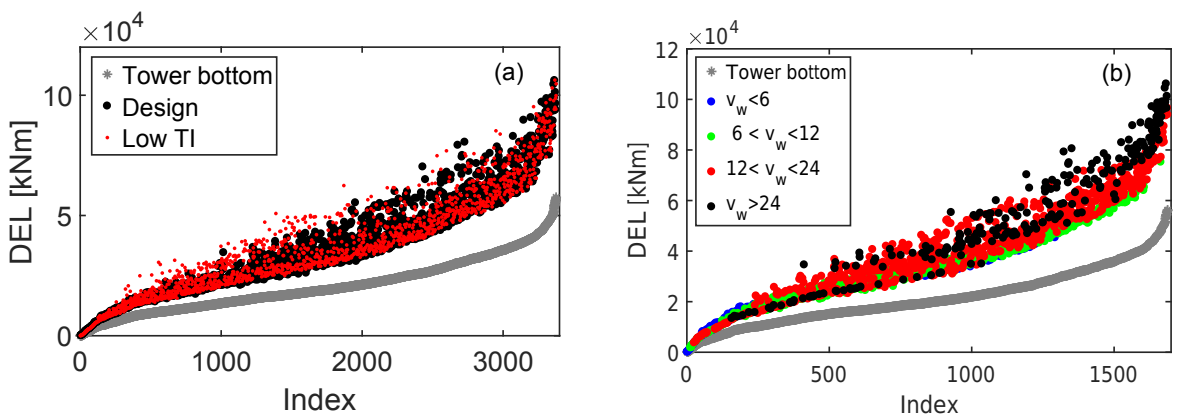

Figure 4. M-DELs as function of ascending T-DELs. (a) M-DELs for design basis (black) and lower turbulence intensity (red). (b) Design basis M-DELs are colored according to the input mean wind speed.

Table 1. Load case combinations presented in groups consisting of mean wind speed $V_{\mathrm{W}}$, significant wave height $H_{\mathrm{S}}$, wave peak period $T_{\mathrm{P}}$, turbulence intensity $T I$, and IEC load case. Each group contains 100-300 simulations of 10 min duration.

\begin{tabular}{lrrrrr}
\hline$V_{\mathrm{W}}$ & $H_{\mathrm{S}}$ & $T_{\mathrm{P}}$ & $\mathrm{TI}$ & $\begin{array}{r}\mathrm{TI} \\
\text { reduced } \\
(\%)\end{array}$ & $\begin{array}{r}\text { IEC } \\
\text { load } \\
\text { case }\end{array}$ \\
\hline $2-4$ & $0.5-1.0$ & $5.0-6.0$ & $15-20$ & $5-6$ & $1.2,6.4$ \\
$5-8$ & $0.5-1.5$ & $5.0-6.0$ & $15-17$ & $4-5$ & $1.2,6.4$ \\
$9-12$ & $1.0-2.0$ & $6.0-7.0$ & $12-15$ & $3-5$ & $1.2,6.4$ \\
$13-16$ & $2.0-3.0$ & $6.5-7.5$ & $10-12$ & $3-4$ & $1.2,6.4$ \\
$17-20$ & $2.5-4.0$ & $7.5-8.5$ & $10-11$ & $3-4$ & $1.2,6.4$ \\
$21-24$ & $4.0-5.0$ & $8.5-9.5$ & $10-11$ & $3-4$ & $1.2,6.4$ \\
$25-28$ & $5.5-6.5$ & $10.0-11.0$ & $10-11$ & $3-4$ & 6.4 \\
$29-32$ & $7.0-8.0$ & $11.5-12.5$ & $10-11$ & $3-4$ & 6.4 \\
\hline
\end{tabular}

of the art. The turbine tower is connected to the monopile with a flanged transition piece.

The extrapolation model is tested in five cases:

1. design basis,

2. extended simulations,

3. design basis with wind speed binning,

4. extended simulations with wind speed binning, and

5. design basis with artificial measurement noise.

M-DELs for the design basis (black) and lower turbulence intensity (red) are plotted in Fig. 4a. The extended simulations follow the same pattern as the original set. In Fig. 4b M-DELs are colored according to their mean wind speed. The lower envelope observed for M-DELs is driven by wind speeds below rated power $\left(12 \mathrm{~m} \mathrm{~s}^{-1}\right)$. The high end of the scatter occurs predominantly for load cases above cut-out wind speed $\left(24 \mathrm{~m} \mathrm{~s}^{-1}\right)$.

The design basis includes 1700 load cases of normal operation and idling with wind speeds from 2 to $32 \mathrm{~m} \mathrm{~s}^{-1}$ and corresponding sea states and turbulence intensity. Wind-wave directionality is considered in $30^{\circ}$ bins including misalignment. The extended simulations include the design basis and additional 1700 load cases with reduced turbulence intensity. Table 1 presents the different load case combinations in groups. Each group contains between 100 and 300 simulations of $10 \mathrm{~min}$ duration with different random realizations (seeds). All wind directions $\left(0-360^{\circ}\right)$ are simulated in bins of $30^{\circ}$ with two sets of yaw errors. In addition, various windwave misalignments between 0 and $90^{\circ}$ are considered for each wind direction.

For the last test case, artificial noise was imposed on the time series of bending moments at tower bottom extracted from the simulation model to represent potential measurement errors from strain sensors. The measurement noise was modeled as white Gaussian noise with zero mean and a signal-to-noise ratio of $40 \mathrm{~dB}$. The procedure of rainflow counting and DEL calculation was performed equally to the previous test cases without artificial noise.

\subsection{Extrapolation results}

Figures 5 and 6 present results of the extrapolation model in the five test cases. Results for the test cases "design basis" $D$, "design basis with wind speed binning" $D_{\text {wind }}$, "extended simulations" $E$, and "extended simulations with wind speed binning" $E_{\text {wind }}$ were obtained with leave-oneout cross-validation. For the test case "design with artificial measurement noise" $D_{\text {noise }}$, the extrapolation model was calibrated with the computed T-DELs and M-DELs without noise. The noise affected "measured" T-DELs were then used to predict corresponding M-DELs.

Figure 5a shows a histogram of 1700 DEL ratios for the case "design basis", where M-DELs are extrapolated as the mean of 1 and 15 neighbors, respectively. It resembles the shape of a normal distribution for one neighbor, while the skewness increases for more neighbors, resulting in some overprediction of the average. Figure $5 \mathrm{~b}$ presents the variance of the M-DEL fractions "predicted value/calculated value" for each test case, using 15 neighbors. Variance of the ratios is below $10 \%$ in all cases. 

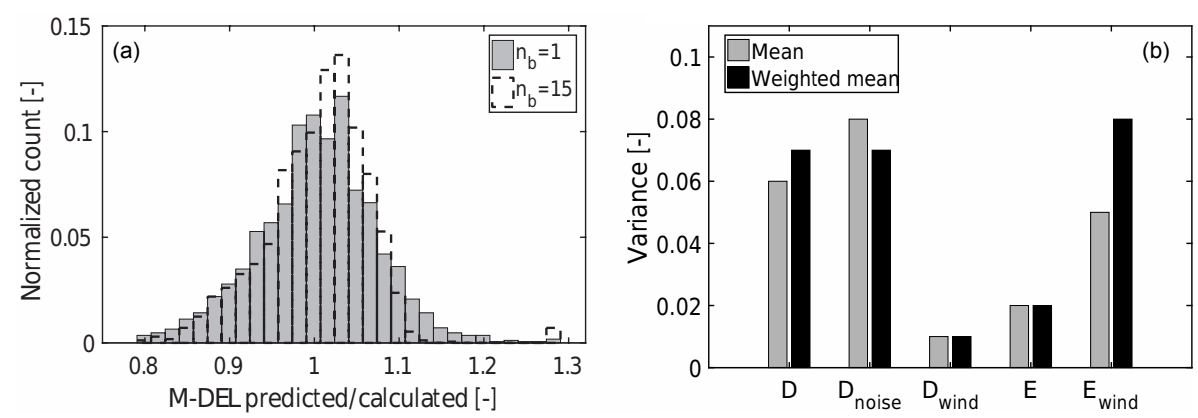

Figure 5. (a) Histogram of DEL ratios for the case "design basis" with 1 and 15 neighbors. (b) Variance of M-DEL ratios for all five test cases using 15 neighbors. The test cases are design basis $D$, design with artificial measurement noise $D_{\text {noise }}$, design basis with wind speed binning $D_{\text {wind }}$, extended simulations $E$, and extended simulations with wind speed binning $E_{\text {wind }}$. The neighbors are taken as mean (grey) or weighted mean (black).
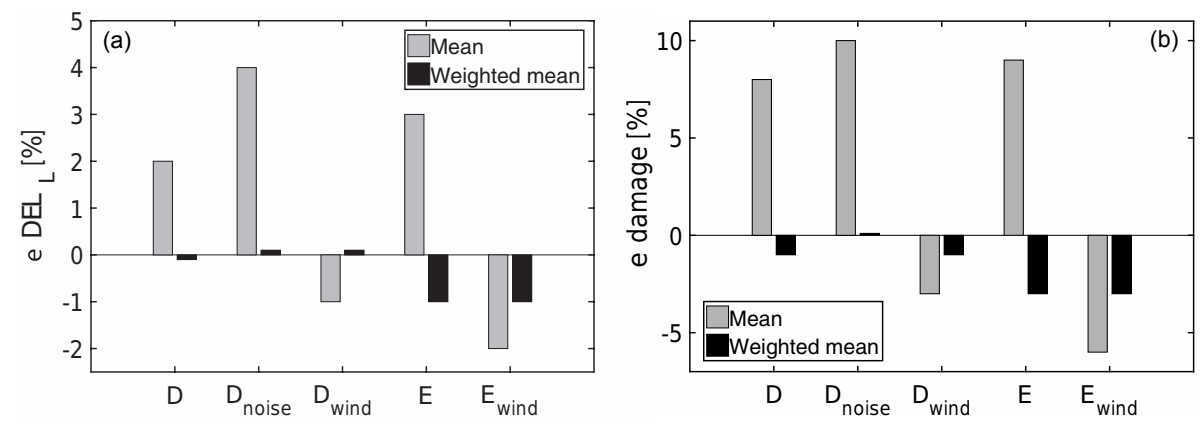

Figure 6. Results for M-DELs with 15 neighbors in five test cases: design basis $D$, design with artificial measurement noise $D_{\text {noise }}$, design basis with wind speed binning $D_{\text {wind }}$, extended simulations $E$, and extended simulations with wind speed binning $E_{\text {wind }}$. The neighbors are taken as mean (grey) or weighted mean (black). (a) Percentage errors between predicted and calculated values of lifetime M-DELs. (b) Percentage errors between predicted and calculated values of lifetime damage.

The percentage errors $e$ between predicted and calculated values for the target parameters, lifetime M-DELs and damage are shown in Fig. 6. Lifetime M-DELs and damage are extrapolated with errors smaller than 3 and $9 \%$ in the simulation environment. The extrapolation error is larger for damage than for lifetime DELs due to exponentiation with the material parameter $m$. Extrapolating DEL ${ }^{m}$ instead of DEL did not yield better results in this study (not shown). Weighting with the probability of occurrence and wind speed binning improves damage extrapolation, leading to errors smaller than 1 and $3 \%$, respectively. Adding artificial noise on the simulated time series of bending moments at tower bottom increased the prediction error of lifetime M-DELs and damage by $1-2 \%$ in this case study.

\subsection{Discussion}

The novel idea for load monitoring is simple and easy to implement. No additional sensors, apart from strain measurements at one level, are needed. As an example, a technical solution would be to install electrical resistance strain gauges at the upper part of the transition piece. The use of four axial strain gauges placed in $90^{\circ}$ intervals around the circum- ferential is recommended. The redundancy of this setup enables the comparison of measurements from opposing strain gauges (compression and tension) to check the level of noise on the data. The sampling rate should be in the range of $20 \mathrm{~Hz}$. The strain data must be calibrated and compensated for temperature. The time series of strain measurements can then be converted into bending stress or bending moments.

Many wind farms already have strain gauges installed at one level of the support structure. Alternatively, a retrofit of the necessary strain gauges is possible in existing wind farms, as no work at submerged parts of the structure is required. In the case of retrofit, there is the potential to link the suggested extrapolation methodology to historical SCADA data (if recorded) in order to estimate the fatigue damage experienced in the past. This requires an additional model to infer DELs from SCADA and environmental conditions. This can be, for instance, a neural network algorithm as suggested by Smolka et al (2014).

Reference is made to Perišić and Tygesen (2014) for a comparison between existing approaches for structural health monitoring and our suggested method. Perišić and Tygesen (2014) compare Kalman-filter-based methods and modal expansion for criteria including computational complexity, 
operation in real time, and structural model complexity. Kalman-filter-based methods have a low computational complexity, use reduced-order FE models and can thus operate in real time. The complexity of structural models and computations for modal-based algorithms is high, resulting in an operation of near-real time (Perišić and Tygesen, 2014). Once the simulation data basis of the methodology presented here is set up, predictions can be performed with almost no computational effort. This also makes it possible to analyze large data sets in retrospect. Algorithms based on artificial intelligence show similar computational performance. These algorithms, however, need sensors at every location for a training period. Perišić and Tygesen (2014) state that Kalmanfilter-based methods and modal expansion perform similarly in terms of accuracy and sensitivity towards measurement noise. Future work with measurement data is needed to evaluate the sensitivity of the proposed methods to measurement noise.

Data transfer and storage of the presented method is efficient as the algorithm works with 10 min values after conversion to DELs. The small costs of this solution make it feasible for application at every turbine in a wind farm. This removes the uncertainty of interpolation between turbines. DELs are calculated for SN curves with one slope here but the method works similarly for bilinear SN curves in correspondence to Cosack (2011). Loads occurring during service life are tracked directly at the sensor location and indirectly at other locations. This enables a comparison with (updated) design load calculations, from which remaining useful lifetime can be derived.

The extrapolation method is exemplarily presented here from tower bottom to mudline. The algorithm was tested for other locations with comparable results (not shown here). The accuracy of the extrapolation method improves the smaller the distance between measurement and predicted location is.

Limitations are that an accurate simulation model is required. Updates are necessary whenever changes of structural properties occur (e.g., change of natural frequency due to scour). Reliability of strain sensors can be affected by temperature and other parameters. The method requires continuous strain monitoring, so degradation of sensors over time might become problematic.

\section{Conclusion and future work}

This paper presents a method to extrapolate load measurements from one location to all hot spots of a monopile. Results are discussed for extrapolation from tower bottom to mudline as an example. We conclude that the correlation between DELs at different locations of the structure offers large potential for low-cost monitoring as only strain measurements at one level are needed. Many offshore wind farms have the necessary sensors and data already available, making the developed method convenient for direct application. First tests show good accuracy of the suggested algorithm, but further validation is necessary. The idea seems very promising and is highly recommended for further development. Future work should address the following:

- Validation with strain measurement data from two locations of a monopile.

- Sensitivity analysis of the extrapolation model to changes in structural properties.

- Detailed study on requirements of data resolution for calculation of DELs, number of simulations for extrapolation model, and measurement duration.

- Integration of freely available SCADA data.

Data availability. No data sets were used in this article.

Competing interests. The authors declare that they have no conflict of interest.

Acknowledgements. We thank Anika Johansen and Daniel Kaufer (Ramboll) for input on monopile design. This project received funding from the European Union's Horizon 2020 research and innovation program through Marie Sklodowska-Curie grant agreement no. 642108 .

Edited by: Gerard J. W. van Bussel

Reviewed by: two anonymous referees

\section{References}

Cosack, N.: Fatigue load monitoring with standard wind turbine signals, PhD thesis, Universität Stuttgart, Germany, 2011.

Devriendt, C., Weijtjens, W., El-Kafafy, M., and De Sitter, G.: Monitoring resonant frequencies and damping values of an offshore wind turbine in parked conditions, IET Renew. Power Gen., 8, 433-441, 2014.

DNVGL: Fatigue design of offshore steel structures, Recommended practice RP-C203, 2016.

Ersdal, G. and Hörnlund, E.: Assessment of offshore structures for life extension, In ASME 2008 27th International Conference on Offshore Mechanics and Arctic Engineering, American Society of Mechanical Engineers, 2008.

Fallais, D. J. M., Voormeeren, S., and Lourens, E.: Vibration-based Identification of Hydrodynamic Loads and System Parameters for Offshore Wind Turbine Support Structures, Energy Procedia, 94, 191-198, https://doi.org/10.1016/j.egypro.2016.09.222, 2016.

Friswell, M. I. and Mottershead, J. E.: Finite Element Model Updating in Structural Dynamics, the Netherlands, Kluwer Academic Publishers, 1995. 
Ho, A. and Mbistrova, A.: The European Offshore Wind Industry Key Trends and Statistics 2016, Report, Wind Europe, Brussels, 2017.

IEC: Wind turbines - part 3: Design requirements for offshore wind turbines, International standard IEC 61400-3, 2009.

Iliopoulos, A., Shirzadeh, R., Weijtjens, W., Guillaume, P., Van Hemelrijck, D., and Devriendt, C.: A modal decomposition and expansion approach for prediction of dynamic responses on a monopile offshore wind turbine using a limited number of vibration sensors, Mech. Syst. Signal Pr., 68, 84-104, https://doi.org/10.1016/j.ymssp.2015.07.016, 2016.

Maes, K., Iliopoulos, A., Weijtjens, W., Devriendt, C., and Lombaert, G.: Dynamic strain estimation for fatigue assessment of an offshore monopile wind turbine using filtering and modal expansion algorithms, Mech. Syst. Signal Pr., 76, 592-611, https://doi.org/10.1016/j.ymssp.2016.01.004, 2016.

Murphy, K. P.: Machine learning: a probabilistic perspective, MIT press, 2012.

Øye, S.: FLEX5 manual, Technical report, Technical University of Denmark, Denmark, 1999.

Passon, P.: Offshore Wind Turbine Foundation Design, $\mathrm{PhD}$ thesis, DTU, Denmark, 2015.

Passon, P. and Branner, K.: Load calculation methods for offshore wind turbine foundations, Ships and Offshore Structures, 9, 433449, https://doi.org/10.1080/17445302.2013.820108, 2014.
Perišić, N. and Tygesen, U. T.: Cost-Effective Load Monitoring Methods for Fatigue Life Estimation of Offshore Platform, in: ASME 2014 33rd International Conference on Ocean, Offshore and Arctic Engineering, American Society of Mechanical Engineers, 2014.

Smolka, U. and Cheng, P. W.: On the design of measurement campaigns for fatigue life monitoring of offshore wind turbines, The Twenty-third International Offshore and Polar Engineering Conference, International Society of Offshore and Polar Engineers, 2013.

Smolka, U., Kaufer, D., and Cheng, P. W.: Are Sea State Measurements Required for Fatigue Load Monitoring of Offshore Wind Turbines?, Journal of Physics: Conference Series, IOP Publishing 555, 012095, https://doi.org/10.1088/17426596/555/1/012095, 2014.

Ziegler, L. and Muskulus, M.: Lifetime extension of offshore wind monopiles: Assessment process and relevance of fatigue crack inspection, 12th EAWE PhD Seminar, DTU Lyngby, Denmark, 2016a.

Ziegler, L. and Muskulus, M.: Fatigue reassessment for lifetime extension of offshore wind monopile substructures, Journal of Physics: Conference Series, IOP Publishing, 753, https://doi.org/10.1088/1742-6596/753/9/092010, 2016b.

Ziegler, L., Gonzalez, E., Rubert, T., Smolka, U., and Melero, J.: Lifetime extension of onshore wind turbines: A review for Germany, Spain, Denmark, and the UK, Renewable and Sustainable Energy Reviews, submitted, 2017. 